\title{
Integration of open source software tools for the in silico design of metabolic pathways using flux balance analysis - an application to the production of recombinant human proteins
}

\section{Ana Sofia Figueiredo*}

\author{
Address: Instituto Gulbenkian de Ciencia, Portugal. \\ Email: Ana Sofia Figueiredo* - sofiafig@igc.gulbenkian.pt \\ * Corresponding author
}

\author{
from BioSysBio: Bioinformatics and Systems Biology Conference \\ Edinburgh, UK, 14-15 July 2005
}

Published: 2 I September 2005

BMC Bioinformatics 2005, 6(Suppl 3):PI I

This study describes the development of a system that calculates the fluxes of a metabolic network. It integrates several open source software tools in order to simulate the behaviour of specific metabolic pathways using Flux Balance Analysis (FBA). This tool receives as input the stoichiometry, the thermodynamic and capacity constraints of the metabolic network, and also an objective function.

The simulation produces predicted values for the fluxes. The metabolic network is represented in SBML $[1,2]$, which is parsed using libSBML [3]. The additional data, in a text file, is parsed using FLEX [4]. This allows the automatic incorporation of the data into a program with GNU lp-solve [5] functions calls. The result is a possible flux distribution that maximises the objective function.

In this work, data from batch fermentation experiments is incorporated in the model. The host system is Escherichia coli BL21, which was engineered to produce a recombinant human protein. Uptake and production rates were calculated using parameter estimation and were incorporated in the model as capacity constraints.

The analysis of the flux distribution was done for the maximisation of ATP production. A discrete time analysis was performed, using the same variables, and assuming a steady state for each time sample. Simulation results show that the induction of protein in the host system decreases the capacity of producing ATP. The response to input variations was studied for variations of $1 \%$ and $10 \%$ at several operating points. The system shows a linear response to these imposed variations.

\section{References}

I. The Systems Biology Markup Language [http://sbml.org/]

2. Hucka M, Finney A, Sauro HM, Bolouri H, Doyle JC, Kitano H, the rest of the SBML Forum: The systems biology markup language (SBML): a medium for representation and exchange of biochemical network models. Bioinformatics 2003, 19:524-531.

3. What is LibSBML? [http://www.sbml.org/software/libsbml]

4. Introduction to Flex [http://www.gnu.org/software/flex/]

5. Solve (mixed integer) linear programming problems [http:I /packages.debian.org/stable/math/lp-solve] 